\title{
Utilizing Conventional and In Situ Electron Microscopy to Gain Insights Into the Release of Silver Nanomaterials in Aquatic Ecosystems
}

\author{
Eric V. Formo ${ }^{1}$, Caroline B. Potterf ${ }^{1}$, Hannah M. Walters ${ }^{1}$, Miaoxin Yang ${ }^{2}$, Raymond R. Unocic ${ }^{3}$, \\ Donovan N. Leonard ${ }^{3}$ and Michelle Pawel $^{3}$ \\ 1. Georgia Electron Microscopy, University of Georgia, Athens, USA. \\ 2. Biomedical Engineering, Georgia Institute of Technology, Atlanta, USA. \\ 3. Center for Nanophase Materials Sciences, Oak Ridge National Laboratory, Oak Ridge, USA.
}

In studying the release of nanostructures into the environment, microscopy as an analytical technique has been historically under-utilized in determining key factors that relate to the aging of these materials [1]. Specifically, much interest to date has been given to studying the exposure of silver nanomaterials into aquatic systems due to their wide array of possible industrial and consumer applications leading to their release [2]. However, the analytical tools utilized previously do not give an entire picture of the fundamental processes of the dissolution of $\mathrm{Ag}(+)$ ions as well as their reduction and growth into larger Ag (0) structures. To this end, we have employed both conventional HR-TEM as well as in situ TEM in order to gain new insights into the fate of Ag nanostructures placed in aquatic ecosystems [3].

Conventional HR-TEM was used to ascertain which exposed crystalline facets are the most environmentally harmful. This was done by taking Ag Nanoparticles (NPs) (Figure 1A) with their exposed $<111>$ and $<100>$ facets and comparing them to the aging profile of Ag Nanocubes (NCs) (Figure 1B) with their large $<100>$ surface facets whilst placing in the aquatic media of pond water, synthetic hard water, and seawater. UV-Vis spectroscopy was employed to find the key moments of sedimentation or dissolution as shown by a decrease in the maximum peak of the UV-Vis spectra and samples for TEM analysis, were taken at those times. Subsequent TEM analysis revealed that the variance in UV-Vis data was caused by an extreme difference in the degradation mechanism of the two opposing nanostructures. We found that NPs undergo sedimentation with the particles aggregating into larger less toxic meso-scale aggregates (Figure 1C). However for Ag NC, the aging simply leads to dissolution (Figure 1D). Further investigation with HR-TEM shows that the root cause of this difference is caused by the Ag $<111>$ facet present in the NPs rapidly undergoes dissolution, followed by subsequent reduction leading to the growth of the larger structures. Whereas the NC with their large exposed $<100>$ faces imbue it with much more stability, greatly slowly the dissolution process, making them much more environmentally robust across all test media [3].

To further study Ag ions released from Ag nanomaterials we employed in situ TEM to gain real-time insights (Figure 2). In particular, we utilized a liquid cell to analyze the beginning stages of Ag structure formation caused by the reduction of Ag ions that are present due to their dissolution from Ag NPs in seawater. Advantageously, beam exposure caused a rapid reduction of the Ag ions allowing us to view their growth upon a faster time scale in comparison to the ex situ experiments previously mentioned. Specifically, we first observed small Ag particles immediately forming (Figure 2A). After 20 seconds the particles increased in size and their morphology begun to change with small 3-4 armed nanostructures that are roughly $400 \mathrm{~nm}$ in diameter being observed (Figure 2B). These armed structures continued to grow and began to have a more dendritic morphology (Figure 2C and $\mathrm{D}$ ). These dendritic structures became the primary morphology in the system attaining a diameter of roughly $2 \mu \mathrm{m}$ after 5 min (Figure 2E). 


\section{References:}

[1] S. Wagner et al, Angew. Chem. Int. Ed. 53 (2014), p. 12398.

[2] T. M. Benn, P. Westerhoff, Environ. Sci. Technol. 42 (2008), p. 4133.

[3] E. V. Formo et al, Environ. Sci. Technol 50 (2016), p. 7082.
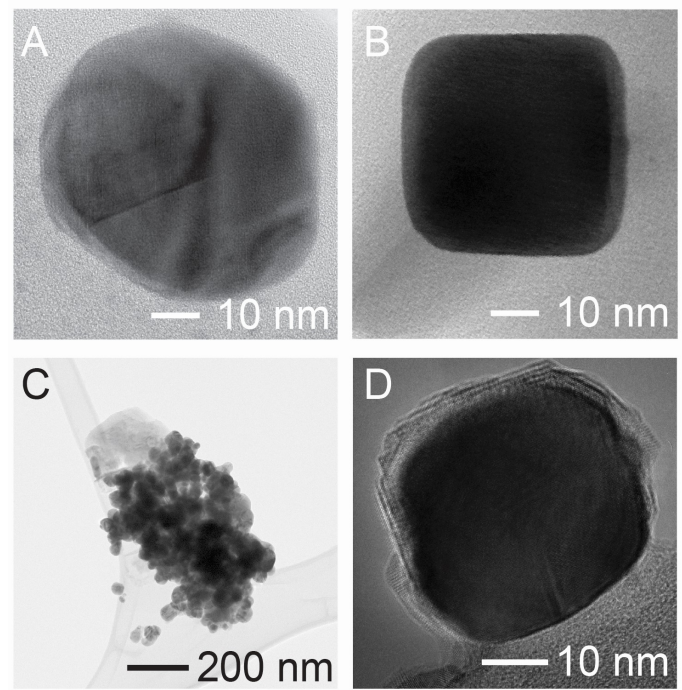

Figure 1. As synthesized Ag nanoparticle (A) and Ag nanocube (B) before being placed in the aquatic media where after four day of aging were removed for TEM analysis which showed, the nanoparticles were heavily aggregated, where as the nanocubes remained largely intact.
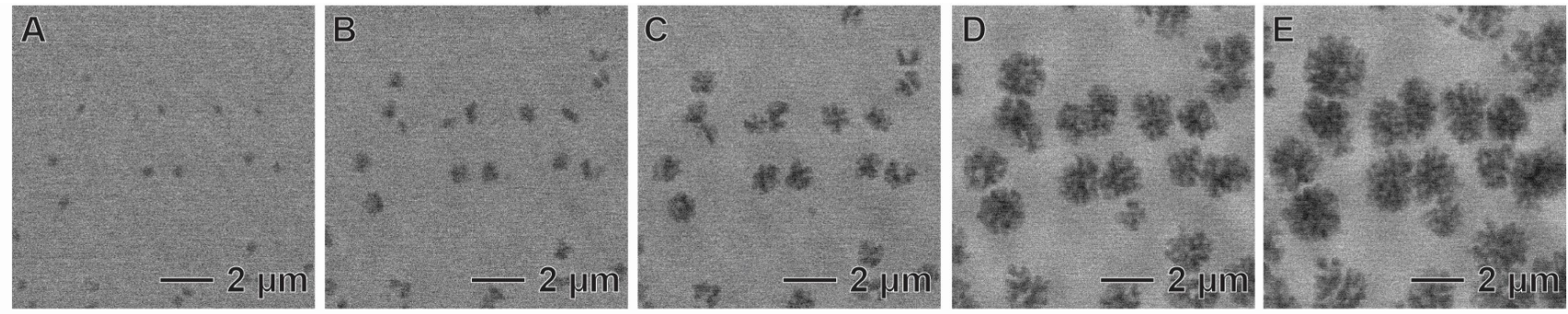

Figure 2. Individual images from an in situ liquid cell TEM experiment at different times in the aging process of initial (A), 20 seconds (B), 40 seconds (C), 2.5 minutes (D), and 5 minutes (E) in which we see the growth of $\mathrm{Ag}$ nanostructures in seawater. 